\title{
Imaging Features of Symptomatic MCA Stenosis in Patients of Different Ages: A Vessel Wall MR Imaging Study
}

\author{
(D) H. Kang, (D). Liang, (D)Y. Zhang, (D) Z. Li, (D)X. Yang, and (D) B. Sui
} - $=$

\begin{abstract}
BACKGROUND AND PURPOSE: The prevalence of intracranial artery stenosis is high in Asian people. This study aimed to investigate whether there are differences in the imaging features of symptomatic MCA stenosis in patients of different ages using vessel wall MR imaging.
\end{abstract}

MATERIALS AND METHODS: We retrospectively reviewed the data of consecutive patients with unilateral MCA stenosis based on a prospectively established vessel wall MR imaging data base between January 2017 and December 2018. According to age, the patients were divided into the young group (18-45 years of age) and the middle-aged and elderly group (older than 45 years of age).

RESULTS: Overall, 131 patients with unilateral MCA stenosis were included (45.8\% in the young group and $54.2 \%$ in the middle-aged and elderly group). Middle-aged and elderly patients had a higher prevalence of hypertension $(P=.01)$ and diabetes $(P=.05)$. The lesion length $(P<.0001)$, proportion of circular involvement $(P=.006)$, and proportion of circular enhancement $(P=.03)$ were higher in the young group than in the middle-aged and elderly group. The analysis of the atherosclerotic subgroup showed that compared with middle-aged and elderly patients, young patients had longer lesions $(P=.002)$. The atherosclerotic-versus-nonatherosclerotic subgroup analysis showed that the maximal wall thickness in the patients with atherosclerosis was larger than that of patients without it $(P=.002)$.

CONCLUSIONS: Compared with the middle-aged and elderly group, young patients with MCA stenosis tended to have longer lesions and more circular wall involvement and circular enhancement, which may indicate the differences in underlying vascular pathophysiologic and developmental mechanisms in symptomatic MCA stenosis.

ABBREVIATIONS: $\mathrm{AS}=$ atherosclerosis; HR VW-MRI = high-resolution vessel wall MR imaging; $M O P=$ middle-aged and elderly; $\mathrm{NWI}=$ normalized wall index; $\mathrm{RI}=$ remodeling index; $\mathrm{SI}=$ signal intensity

schemic stroke is an important cause of death and disability in adults. The incidence rate of ischemic stroke in young people has increased by $40 \%$ in the 2020 s compared with the 2010 s. $^{1,2}$

Received February 23, 2021; accepted after revision June 11.

From the Beijing Neurosurgical Institute (H.K., Y.Z., X.Y.), Beijing Tiantan Hospital, Capital Medical University, Beijing, China; Shijingshan Teaching Hospital of Capital Medical University (J.L.), Beijing Shijingshan Hospital, Beijing, China; and Tiantan Neuroimaging Center for Excellence (Z.L., B.S.), China National Clinical Research Center for Neurological Diseases, Beijing Tiantan Hospital, Capital Medical University, Beijing, China.

H. Kang and J. Liang share first authorship.

This work was supported by Beijing Municipal Natural Science Foundation (grant No. 7212028)

Please address correspondence to Binbin Sui, MD, Tiantan Neuroimaging Center of Excellence, Beijing Tiantan Hospital, Capital Medical University, Beijing, China, No.119 South Fourth Ring West Rd, Fengtai District, Beijing, 100050, China; e-mail: reneesui@163.com

- Indicates open access to non-subscribers at www.ajnr.org

Indicates article with online supplemental data.

http://dx.doi.org/10.3174/ajnr.A7268
Intracranial artery stenosis is an important cause of ischemic stroke and is more commonly found in Asian people. Although atherosclerosis (AS) is still considered the most common reason, the etiology of intracranial artery stenosis, especially in young people, is more complex and diverse, often making the clinical diagnosis difficult and uncertain.

Traditional imaging technologies, including CT angiography, MR angiography, and digital subtraction angiography, can provide information only on lumen stenosis, which has limited value in the etiologic differential diagnosis. In recent years, highresolution vessel wall MR imaging (HR VW-MR imaging) has been increasingly applied in clinical practice. The technique has proved to be an optimal and reliable method to display intracranial vessel wall features. By directly visualizing the structure of the vessel wall and identifying the characteristics of lesions, HR-VW-MR imaging has shown great potential and application prospects in the evaluation of patients with intracranial artery stenosis. ${ }^{3-9}$ It has been proposed that different vessel wall imaging 
features can be found in various diseases, including dissection, vasculitis, and Moyamoya disease, ${ }^{10-12}$ and they can provide important information for clinical assessment.

Although it was reported that AS is still the most common cause of intracranial artery stenosis in young patients, multiple etiologies other than AS should be considered. It is generally believed that AS is more commonly found in older patients. With similar findings of artery stenosis by conventional MRA, different HR VW-MR imaging manifestations due to various stenosis mechanisms may be found in young and elderly groups. One previous study has shown that concentric wall thickening and enhancement on HR VW-MR imaging were found more commonly in patients younger than 35 years of age than in patients $35-45$ years of age. ${ }^{13}$ However, it is still unclear whether there are different HR VW-MR imaging features in patients of different ages, and there is no comparison study of HR VW-MR imaging features between young and middle-aged and elderly (MOP) patients. In this study, using HR VW-MR imaging, we sought to identify differences in the imaging features of stenosed MCA lesions of atherosclerosis and nonatherosclerotic disease in patients of different ages.

\section{MATERIALS AND METHODS \\ Participants}

We retrospectively reviewed the data of consecutive patients with unilateral MCA stenosis based on a prospectively established HR VW-MR imaging data base between January 2017 and December 2018. Our ethics committee approved this retrospective study, and the requirement for written informed consent was waived due to its retrospective nature. Consecutive patients with ipsilateral MCA stenosis were included. The inclusion criteria were as follows: 1) symptomatic patients who had an ischemic stroke or TIA, 2) unilateral MCA stenosis confirmed by CT angiography or MRA, or 3) the stenosed MCA ipsilateral to the ischemic lesion or responsible for the TIA and defined as the culprit (index) vessel by the neurologist and neuroradiologist. Patients were excluded for the following reasons: 1) definite cardiogenic embolism, such as a history of atrial fibrillation confirmed by electrocardiogram or a Holter monitor within 1 month or a recent history of myocardial infarction, atrial septal defect, or left atrial/ventricular thrombosis confirmed by echocardiography; 2) extracranial carotid stenosis of $>50 \% ;{ }^{14}$ unstable plaques of the extracranial carotid artery that met 3 of the following 4 criteria: stenosis $>70 \%$, mainly hypoechoic on ultrasound images, superficial irregularity, and ulceration; 3) definite Moyamoya disease; 4) ipsilateral or bilateral MCA occlusion or bilateral MCA stenosis; or 5) poor MR image quality or lack of clinical data. The clinical data (including age, sex, smoking history, hypertension, diabetes, hyperlipidemia, and homocysteine) and the HR VW-MR imaging characteristics were reviewed and analyzed. According to age, the patients were divided into the young group (18-45 years of age) and the MOP group (older than 45 years of age).

\section{MR Imaging Protocol}

MR imaging was performed using 3T MR imaging scanners (Trio Tim, Siemens; Discovery 750, GE Healthcare; and Ingenia CX, Philips Healthcare) with a 32-channel head coil. The MR imaging protocol included 3D TOF-MRA, 3D T1WI (sampling perfection with application-optimized contrasts by using different flip angle evolution [SPACE sequence, Siemens]/Cube [GE Healthcare]/volume isotropic turbo spin-echo acquisition [VISTA]), 3D T2/PDWI proton-density weighted imaging (PDWI) (SPACE/CUBE/VISTA), 3D T1 MPRAGE, and contrast-enhanced 3D T1WI (SPACE/ CUBE/VISTA). Postcontrast T1-weighted images were acquired 3 minutes after gadolinium injection $(0.1 \mathrm{mmol} / \mathrm{kg}$ of gadopentetate dimeglumine, Magnevist; Bayer HealthCare Pharmaceuticals) using parameters identical to those of the precontrast T1-weighted images. The parameters of the imaging sequences are listed in the Online Supplemental Data.

\section{Image Analysis}

All MR images were transferred to a dedicated postprocessing workstation for analysis with Vessel Explorer software (Version 1.1; TsingHua Imaging Co). HR VW-MR imaging data were analyzed by 2 experienced neuroradiologists (J.L. and B.S.) independently, who were blinded to the clinical data. Differences between the 2 observers were solved by consensus.

The vessel centerline of the MCA was extracted automatically on TOF-MRA and copied to the precontrast 3D T1-weighted images. Then, oblique sagittal precontrast T1-weighted images were reconstructed perpendicular to the M1 segment of the relevant MCA with a reconstructed section thickness of $0.7 \mathrm{~mm}$. The analysis plane was set as the maximal lumen narrowing site. The reference site was defined as the nearest lesion-free segment proximal or distal to the lumen narrowing site. Precontrast T1weighted images were used for the main analysis, including the measurements of lumen and wall thickness at the analysis and reference planes, assessment of the wall thickening and involvement pattern, remodeling patterns, and the surface morphology. A 3D T1 MPRAGE sequence was used to identify intralesion hemorrhages. Postcontrast T1-weighted images were used for the enhancement assessment. 3D T2-weighted and proton-density weighted images were used as supplementary images if there was obscurity of the vessel wall on T1-weighted images.

The maximum wall thickness and lesion length were measured in the circumferential plane and along the longitudinal axis of the artery, respectively (Fig 1A). The degree of stenosis was calculated as follows: degree of stenosis $=(1-$ Lumen Area at the Maximal Lumen Narrowing Site/Reference Lumen Area) $\times 100 \%{ }^{15,16}$ The normalized wall index (NWI) was calculated as follows: NWI = Wall Area/(Lumen Area + Wall Area). ${ }^{17}$ The wall thickening, involvement pattern, and remodeling patterns of the index MCA were analyzed on precontrast T1-weighted images, as was the surface morphology. In the circumferential plane, the wall of the MCA was divided into 4 quadrants: namely, the superior wall, the inferior wall, the ventral wall, and the dorsal wall (Fig $1 B$ ); and circular involvement was defined as lesions involving all 4 quadrants. The pattern of wall thickening was classified as concentric or eccentric (Fig 1C, $-D$ ). Concentric stenosis was identified if the thinnest part of the vessel wall was estimated to have a thickness no less than $50 \%$ of the thickest point on all image slices or a stenosis without wall thickening. ${ }^{6}$ Eccentric stenosis was diagnosed if the thinnest part of the vessel wall was estimated to have a thickness of $<50 \%$ of the thickest point. ${ }^{15}$ The remodeling index (RI) 


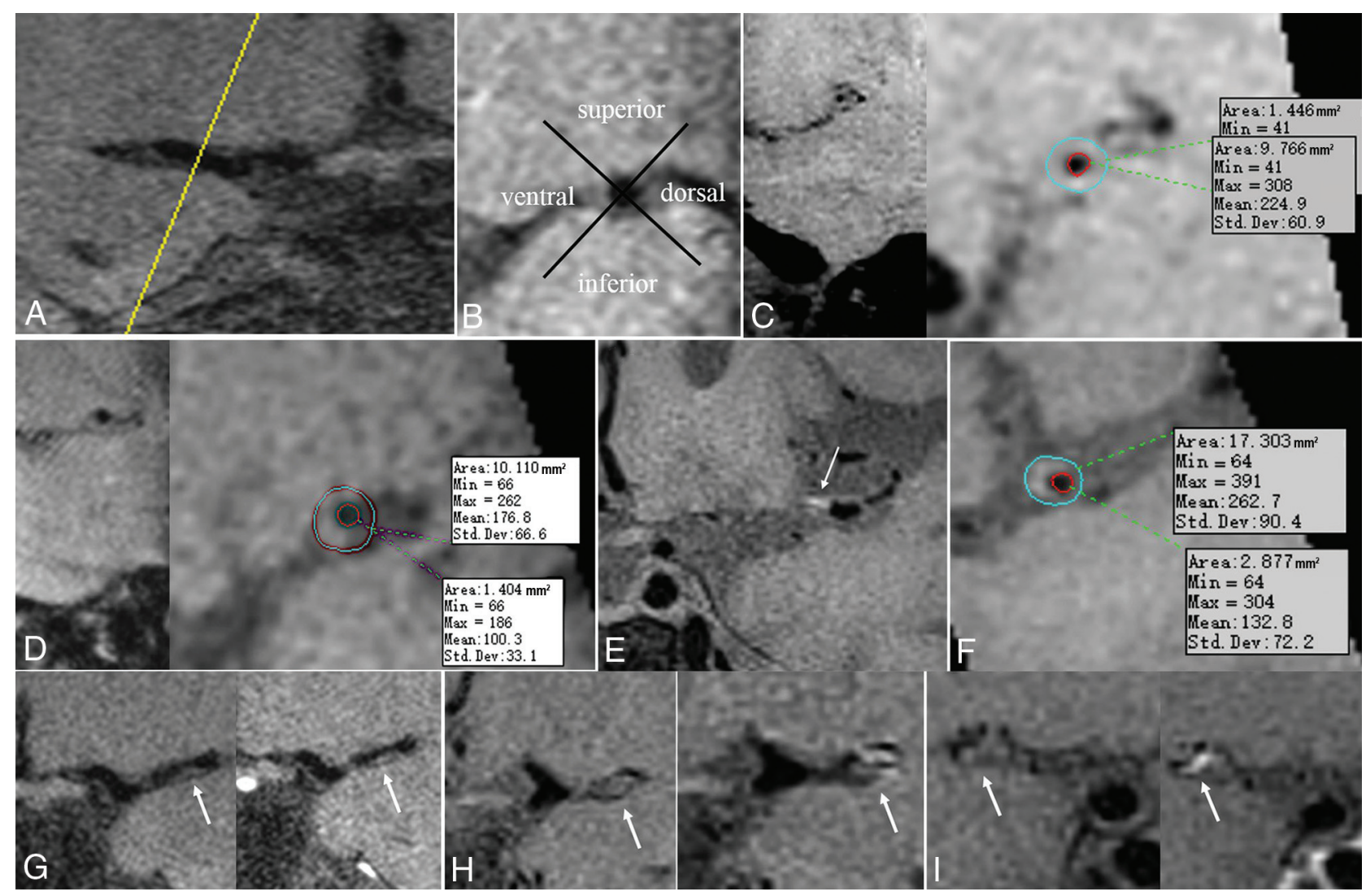

FIG 1. A, Normal MCA. B, Enlarged view perpendicular to the lumen section. The wall of the MCA is divided into 4 quadrants: namely, the upper wall, the lower wall, the ventral wall, and the dorsal wall. C, Annular thickening of the vessel wall, ie, concentricity. D, Eccentric thickening of the vessel wall. E, Local $\mathrm{Tl}$ hyperintensity in the M1 segment of the left MCA. $F$, An enlarged cross-section of the blood vessel at the lesion shows eccentric wall thickening and T1 hyperintensity. Degree of lesion enhancement: grade $0(G)$; grade I (H); grade II (I). Min indicates minimum; Max, maximum, Std. Dev., standard deviation.

was calculated as follows: RI $=$ Lumen Area at the Maximal Lumen Narrowing Site / Reference Lumen Area. RI $\geq 1.05$ was considered positive remodeling, $\mathrm{RI} \leq 0.95$ was considered negative remodeling, and $0.95<\mathrm{RI}<1.05$ nonremodeling. ${ }^{18}$

Intralesion hemorrhage ${ }^{19}$ was defined as high signal intensity (SI) in MCA lesions on 3D T1 MPRAGE images, with an SI of $>150 \%$ of the adjacent brain parenchyma (Fig $1 E,-F) .{ }^{20}$ On postcontrast T1-weighted images, the degree of lesion enhancement was defined using the following grading criteria: grade 0 , the degree of enhancement less than or equal to that of the adjacent normal arterial wall (Fig $1 G$ ); grade I, the degree of enhancement greater than grade 0 but lower than that of the pituitary funnel (Fig $1 H$ ); and grade II, the degree of enhancement greater than or equal to that of pituitary funnel enhancement (Fig 1I). ${ }^{21}$

Maaijwee et $\mathrm{al}^{22}$ reported that traditional vascular risk factors in young adults, especially in patients between 35 and 50 years of age, have increased in prevalence, indicating a sharp rise. Therefore, we further conducted a subgroup analysis by dividing patients into the older than 35-year group and the 35year-or-younger group. In addition, to clarify the difference in the characteristics of lesions between young and MOP patients with different degrees of stenosis, patients with stenosis of $\geq 50 \%$ and patients with stenosis of $<50 \%$ were classified into different subgroups. We also performed subgroup analysis of confirmed atherosclerotic plaque cases. Atherosclerotic lesions were defined according to the following diagnostic criteria: The clinical diagnosis of AS required $\geq 2$ vascular risk factors (hypertension, hyperlipidemia, diabetes mellitus, obesity, coronary artery disease, men older than 50 years of age, and women older than 60 years of age) and failure to meet the clinical criteria for CNS vasculitis or reversible cerebral vasoconstriction syndrome. ${ }^{23}$ The typical HR-VW-MR imaging diagnosis of AS is based on eccentric, irregular, heterogeneous wall thickening with vessel wall enhancement and T2-weighted hyperintensity. ${ }^{24}$ The diagnosis was confirmed by consensus of the neurologist, neurointerventional surgeon, and neuroradiologist. The clinical and imaging features of MCA AS were compared between young and MOP patients. The clinical and imaging features of intracranial atherosclerosis versus nonatherosclerosis were compared in the young group and the middle-aged and older group.

\section{Statistical Analysis}

The Statistical Package for the Social Sciences (SPSS 25.0; IBM) was used to analyze the differences between the 2 groups. The intraclass correlation coefficient was calculated to find the intraobserver and interobserver reproducibility in the measurements of high SI, vessel wall area, and luminal area. Continuous variables are expressed as mean (SD), and categoric variables were analyzed using the Pearson $\chi^{2}$ test or Fisher exact test. 


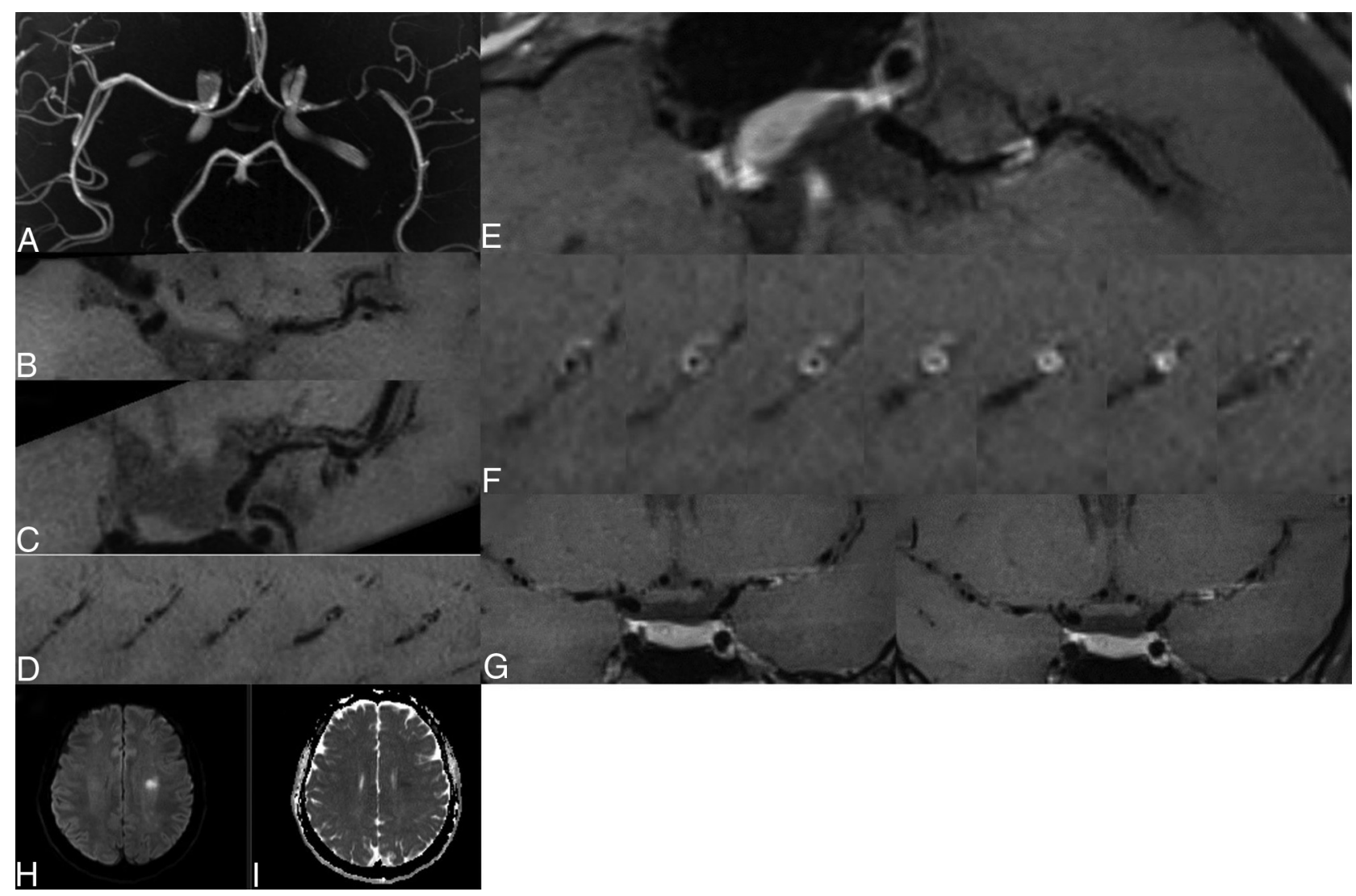

FIG 2. A 37-year-old man. Left MCA stenosis is shown on a 3D TOF-MRA MIP image (A). HR-VW-MR imaging shows an isointense signal lesion on precontrast $\Pi$ images at the stenosed segment of the left MCA, with an irregular surface $(B$ and $C$ ). Oblique serial sagittal images demonstrate a concentric wall thickening lesion with circular wall involvement $(D)$. Postcontrast $\Pi$ images show intense (grade II) concentric enhancement of the lesion $(E-G)$. DWI shows a small patchy acute ischemic lesion in the left centrum semiovale, with restricted diffusion displayed $(H$ and $I)$.

Continuous variables were compared between the 2 groups by the independent-samples $t$ test or the Mann-Whitney $U$ test. The significance threshold was set at $P<.05$.

\section{RESULTS}

\section{Demographic Data of Young and MOP Groups}

In total, 131 patients with unilateral MCA stenosis were included from January 2017 to December 2018. The mean age of the patients was 47.1 (SD, 12.5) years, and $16.8 \%$ (22/131) were women. The young group included 60 patients (45.8\%), and the MOP group included 71 patients (54.2\%). The young group included 27 patients (45.0\%) with TIA and 33 patients (55\%) with infarction, and the MOP group included 20 patients (28.2\%) with TIA and 51 patients $(71.8 \%)$ with infarction. The median interval time between symptom onset and HR VW-MR imaging was 4.3 (SD, 3.3) days. One hundred and eleven (84.7\%) patients had atherosclerotic disease, and 20 (15.3\%) had nonatherosclerotic disease.

\section{Intraobserver and Interobserver Reproducibility for Imaging Analysis}

The intraobserver and interobserver reproducibility of measurements of high SI were 0.870 (95\% CI, 0.773-0.969) and 0.936 (95\% CI, 0.860-1.000), respectively. The intraobserver and interobserver reproducibility of measurements of the wall area were 0.854 (95\% CI, 0.744-0.964) and 0.920 (95\% CI, 0.838-1.000), respectively. The intraobserver and interobserver reproducibility of measurements of the luminal area were 0.838 (95\% CI, 0.731$0.946)$ and 0.918 (95\% CI, 0.846-0.990), respectively.

\section{Comparison of Clinical and Imaging Features between the Young and MOP Groups}

The clinical and imaging features were compared between the young and MOP groups in the Online Supplemental Data. MOP patients had a higher prevalence of hypertension (70.4\% versus $41.7 \%, P=.01)$ and diabetes $(29.6 \%$ versus $15.0 \%, P=.05)$ than young patients. The proportions of circular wall involvement (40.0\% versus $19.7 \%, P=.006)$ and circular enhancement $(68.1 \%$ versus $45.5 \%, P=.03$ ) were higher in the young group than in the MOP group (Figs 2 and 3). Lesions were significantly longer in the young group than in the MOP group (8.47 versus $6.23 \mathrm{~mm}$, $P<.0001)$. The proportion of eccentric thickening was slightly higher in the MOP group (85.9\%) than in young patients (80.0\%), but this difference was not statistically significant $(P=$ .37). No significant differences were found in other parameters between the 2 groups.

\section{Comparison of Clinical and Imaging Features of Atherosclerotic Plaques between Young and MOP Patients}

In total, 111 cases $(84.7 \%)$ were confirmed as peri-interventional plaques (47 cases in the young group and 64 cases in the MOP 


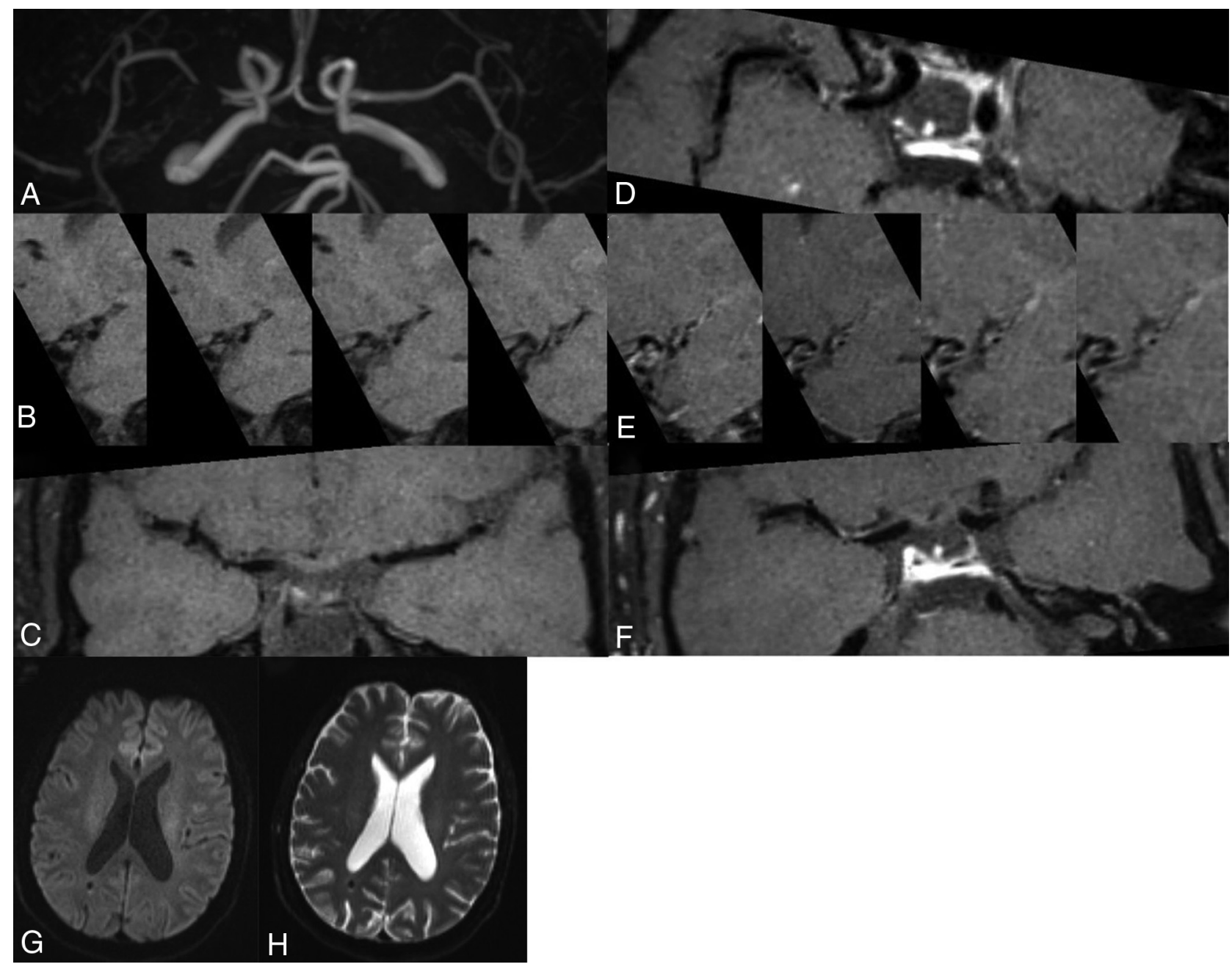

FIG 3. A 37-year-old woman. Right MCA stenosis is shown on a 3D TOF-MRA MIP image (A). HR-VW-MR imaging shows an isointense signal lesion on precontrast $\mathrm{T} 1$ images at the stenosed segment of the right MCA, with a relatively regular surface $(B)$. Oblique sagittal serial images demonstrate an eccentric wall thickening lesion with mainly ventral and superior wall involvement (C). Postcontrast $\mathrm{Tl}$ images show moderate (grade I) eccentric enhancement of the lesion $(D-F)$. DWI shows that there was no abnormally restricted lesion (G-H).

group) (Online Supplemental Data). The subgroup analysis showed that MOP patients had a higher prevalence $(75.0 \%$ versus $44.7 \%$, $P=.001)$ of hypertension than young patients. Lesions were longer in the young group than in the MOP group (8.25 versus $6.28 \mathrm{~mm}$, $P=.002)$. The prevalence of intraplaque hemorrhage was higher in the MOP group (20.3\%) than in the young group (44.7\%), but no significant difference was found between two groups $(P=.09)$.

\section{Comparison of Intracranial Atherosclerotic versus Non- Atherosclerotic Disease in the Young Group and Middle- Aged and Older Group}

The atherosclerosis-versus-nonatherosclerosis subgroup analysis only showed that the wall maximal thickness in the patients with AS was larger than that of patients without atherosclerosis (1.72 versus $1.42 \mathrm{~mm}, P=.002$ ). The patients with AS had a higher prevalence of hypertension $(62.2 \%$ versus $30.0 \%, P=$ $.007)$, diabetes (26.1\% versus $5.0 \%, P=.04)$, hyperlipidemia (63.1\% versus $35.3 \%, P=.02)$, and smoking $(78.4 \%$ versus $50.0 \%, P=.008$ ) than the patients without it (Online Supplemental Data).

\section{Comparison of Clinical and Imaging Features in Different Age/Stenosis Subgroups}

In our subgroup analysis of patients with a degree of stenosis of $>50 \%$, the rates of hypertension $(75.8 \%$ versus $43.3 \%, P=$ .009 ) and diabetes (30.3\% versus $10.0 \%, P=.05)$ were higher in the MOP group than in the young group, while the lesions were longer in the young group than in the MOP group (8.90 versus $6.57 \mathrm{~mm}, P=.002$ ) (Online Supplemental Data).

In our subgroup analysis of patients with a degree of stenosis of $<50 \%$, the rate of hypertension ( $65.8 \%$ versus $40.0 \%, P=.03$ ) and the degree of stenosis (34.7\% versus $26.6 \%, P=.04$ ) were higher in the MOP group than in the young group. Moreover, the lesions were longer in the young group than in the MOP group (8.40 versus $5.95 \mathrm{~mm}, P=.02$ ) (Online Supplemental Data).

\section{Comparison of Clinical and Imaging Features between Patients 35 Years of Age or Younger and Patients 35-45 Years of Age}

Our subgroup analysis by age within the young group revealed that patients 35 years of age or younger had a smaller proportion 
of constrictive remodeling (36.0\% versus $71.4 \%, P=.02)$, less circular wall involvement (20.0\% versus $54.3 \%, P=.005)$, and a higher remodeling ratio (1.05 versus $0.85, P=.004)$ than patients 35-45 years of age (Online Supplemental Data).

\section{DISCUSSION}

In recent years, the incidence of stroke in young adults has markedly increased. ${ }^{20}$ Approximately $10 \%$ of ischemic strokes occur in young patients. ${ }^{22}$ The risk factors and etiologic features of ischemic stroke in young adults are considered very different from those of older patients with stroke with traditional vascular risk factors such as hypertension, hypercholesterolemia, diabetes mellitus, and obesity. ${ }^{25}$ One study reported that the high incidence of traditional vascular risk factors among young people showed the same trend as the increasing incidence of ischemic stroke. ${ }^{20}$ Our results showed that the MOP group had a higher prevalence of hypertension $(P=.01)$ and diabetes $(P=.05)$ than the young patients. The atherosclerosis-versus-nonatherosclerosis subgroup analysis showed that the patients with AS had a higher prevalence of hypertension $(P=.007)$, diabetes $(P=.04)$, hyperlipidemia $(P=.02)$, and smoking $(P=.008)$ than the patients without atherosclerosis. No significant difference was found between people 35 years of age and younger and people 35-45 years of age in these traditional risk factors $(P>.05)$. These findings indicate that traditional risk factors are still mainly prevalent among elderly individuals with atherosclerosis. However, only the incidence of diabetes was collected in the current study. Uncontrolled diabetes is thought to be an important contributor to intracranial plaque. It is necessary to include the analysis of hemoglobin A1C in future studies.

In our subgroup analysis of people with $<50 \%$ stenosis, the degree of stenosis was higher in the MOP group than in the young group (34.7\% versus $26.6 \%, P=.04$ ). This finding might suggest that in cases of mild stenosis, the progression and degree of MCA stenosis in MOP patients may be faster and more severe due to the effects of traditional risk factors. The stenosis degree was $<50 \%$ in 68 patients (30 in the young group, 38 in the MOP group), which suggests that mild and moderate stenosis can be symptomatic. Along with stenosis degree, MCA lesion features on HR VW-MR imaging should considered when assessing MCA stenosis.

HR VW-MR imaging plays a very important role in the evaluation of intracranial artery stenosis. Many features of vessel wall lesions, including the wall thickening morphology, surface status, lesion burden, and the degree and pattern of lesion enhancement, can be analyzed. These detailed features of the involved arterial wall are helpful to establish diagnoses and differential diagnoses of vascular diseases, as well as to evaluate the severity and stability of the lesions. In the current study, we did not find a statistically significant difference in NWI, wall thickening pattern, remodeling pattern, wall maximal thickness, or surface morphology between young and MOP patients (all $P>.05$ ). These results might indicate that these parameters were similar in symptomatic MCA stenosis irrespective of age. However, we found that the wall maximal thickness of the patients with AS was larger than that of the patients without it $(P=.002)$. One possible reason is that many patients with atherosclerosis have eccentric thickening of the vessel wall, so the maximum thickness of the vessel wall is larger than that of the nonatherosclerotic vessel wall.
Eccentric wall thickening was found more often in patients 35 years of age and younger than in patients 35-45 years of age ( $88.0 \%$ versus $74.3 \%, P=.19$ ), and the patients 35 years of age and younger had a lower proportion of constrictive remodeling than patients $35-45$ years of age $(36.0 \%$ versus $71.4 \%, P=.02)$. These results were not in agreement with previous studies, in which concentric wall lesions were more frequently reported in patients younger than 35 years of age. ${ }^{6,23,26,27}$ This difference could be explained by the higher incidence of CNS vasculitis in younger patients in those studies. Smooth, homogeneous, concentric arterial wall thickening and enhancement on HR-VWMR imaging are considered features of CNS vasculitis. However, previous studies have shown that vasculitis sometimes also results in eccentric wall abnormality. ${ }^{28,29}$ This makes the diagnosis of CNS vasculitis more difficult in clinical practice. Our results might indicate that with multiple different risk factors, concentric wall thickening, whether due to vasculitis or not, could be present in patients 35-45 years of age. The incidence of vasculitis in different age groups needs to be confirmed in future studies.

Wall enhancement reflects an inflammatory reaction or the increased permeability of the endothelium, with contrast leakage from the lumen into the arterial wall. Obvious enhancement often presents in the active or unstable phase of the disease. The enhancement degree was not significantly different between different age groups. Because all patients in this study were symptomatic, it could be expected that more than half of them had intense enhancement (grade II) in both the young and MOP groups. The enhancement pattern is another factor that may be related to the vascular physiopathologic mechanism. Circular enhancement was more prevalent in the young group than in the MOP group (68.1\% versus $45.5 \%, P=.03$ ). This result could be expected because circular involvement was also more prevalent in young patients. Whether circular wall involvement and enhancement can be taken as a feature of non-AS vascular disease or whether a larger circumferential burden is required should be further investigated.

One interesting result of our study is that the lesions were significantly longer in the young group than in the MOP group (8.47 versus $6.23 \mathrm{~mm}, P<.0001$ ), while the NWI was not found to be significantly different between the 2 groups. Similar results were also found in subgroups with different degrees of stenosis. Most of the previous HR VW-MR imaging studies have focused on circumferential plane analysis of intracranial artery lesions, while less attention has been paid to the length of the lesion. However, lesion length is an important factor that reflects the longitudinal burden of the lesions, which might affect the distal blood supply of the stenosed artery. One possible explanation for this phenomenon might be the different developmental mechanisms in younger-versus-MOP patients. The anatomic and pathologic factors related to MCA lesion length remain unclear. Younger patients with a degree of wall thickening and stenosis similar to those in older patients might need longer involvement of the MCA to cause clinical symptoms. Another reason for the lesion length difference is the various possible diseases in younger patients. Arterial dissection, for example, could involve a longer lesion length than AS disease. Intracranial artery dissection is still considered relatively rare but is an important cause of intracranial 
stenosis and ischemic stroke, especially in young adults. ${ }^{30,31}$ Patients younger than 45 years of age with intracranial artery dissections have approximately $20 \%$ of strokes and $2 \%$ of all ischemic strokes. ${ }^{22}$ The differences and significance of lesion length in various artery diseases should be investigated in future studies.

Our study included an AS subgroup analysis between young and symptomatic MOP patients. The young patients had a lower prevalence of hypertension $(44.7 \%$ versus $75.0 \%, P=.001)$ and longer plaque length ( 8.25 versus $6.28 \mathrm{~mm}, P=.002$ ) than the MOP patients. The difference between hypertension prevalences indicated that early AS in young patients might be caused by various risk factors. The plaque length results showed a larger longitudinal burden of the involved MCA in young patients. They also suggested that the underlying mechanisms might be different for AS plaques between young and symptomatic MOP patients. Although there are similar medical treatment strategies for AS stenosis, it is worth studying whether this imaging feature difference is related to different outcomes in patients of different ages. Moreover, whether plaque length influences the effect of angioplasty should also be investigated. Further longitudinal studies will provide more valuable information about the clinical significance of this feature.

There are some limitations to our study. First, the retrospective design of this single-center study might have led to selection bias. Some results differed from previous findings, which might be due to the differences between the patients in different studies. The proportion of male patients in this study was relatively high, limiting its generalizability. Second, the imaging data were obtained from 3 different machines, so it was difficult to avoid vendor effects when performing parameter analysis, especially for the measurements of maximal wall thickness and lesion length. Another limitation of the study is the lack of follow-up data, which would be very helpful in the differential diagnosis of various diseases that are difficult to diagnose. Additionally, the dynamic change in vessel wall features would help us to understand the pathologic mechanisms of different vascular diseases.

\section{CONCLUSIONS}

Compared with the MOP group, young patients had longer lesions, more circular wall involvement, and more circular enhancement, which may result from the various vascular physiopathologic mechanisms in symptomatic patients of different ages with MCA stenosis.

\section{REFERENCES}

1. Bejot Y, Bailly H, Durier J, et al. Epidemiology of stroke in Europe and trends for the 21st century. Presse Med 2016;45:e391-98 CrossRef Medline

2. Feigin VL, Roth GA, Naghavi MG, et al; Global Burden of Diseases, Injuries and Risk Factors Study 2013 and Stroke Experts Writing Group. Global burden of stroke and risk factors in 188 countries, during 1990-2013: a systematic analysis for the global burden of disease study 2013. Lancet Neurol 2016;15:913-24 PMC CrossRef Medline

3. Bodle JD, Feldmann E, Swartz RH, et al. High-resolution magnetic resonance imaging: an emerging tool for evaluating intracranial arterial disease. Stroke 2013;44:287-92 CrossRef Medline
4. Li ML, Xu WH, Song L, et al. Atherosclerosis of middle cerebral artery: evaluation with high-resolution MR imaging at 3T. Atherosclerosis 2009;204:447-52 CrossRef Medline

5. Majidi S, Sein J, Watanabe M, et al. Intracranial-derived atherosclerosis assessment: an in vitro comparison between virtual histology by intravascular ultrasonography, 7T MRI, and histopathologic findings. AJNR Am J Neuroradiol 2013;34:2259-64 CrossRef Medline

6. Swartz RH, Bhuta SS, Farb RI, et al. Intracranial arterial wall imaging using high-resolution 3-Tesla contrast-enhanced MRI. Neurology 2009;72:627-34 CrossRef Medline

7. Turan TN, Rumboldt Z, Granholm AC, et al. Intracranial atherosclerosis: correlation between in-vivo $3 \mathrm{~T}$ high resolution MRI and pathology. Atherosclerosis 2014;237:460-63 CrossRef Medline

8. Chen XY, Wong KS, Lam WW, et al. High signal on T1 sequence of magnetic resonance imaging confirmed to be intraplaque haemorrhage by histology in middle cerebral artery. Int J Stroke 2014;9:E19 CrossRef Medline

9. van der Kolk AG, Zwanenburg JJ, Denswil NP, et al. Imaging the intracranial atherosclerotic vessel wall using 7T MRI: initial comparison with histopathology. AJNR Am J Neuroradiol 2015;36:694-701 CrossRef Medline

10. Nakamura $Y$, Yamaguchi $Y$, Makita N, et al. Clinical and radiological characteristics of intracranial artery dissection using recently proposed diagnostic criteria. J Stroke Cerebrovasc Dis 2019;28:16911702 CrossRef Medline

11. Mandell DM, Mossa-Basha M, Qiao Y, et al; Vessel Wall Imaging Study Group of the American Society of Neuroradiology. Intracranial vessel wall MRI: principles and expert consensus recommendations of the American Society of Neuroradiology. AJNR Am J Neuroradiol 2017;38:218-29 CrossRef Medline

12. Ryoo S, Cha J, Kim SJ, et al. High-resolution magnetic resonance wall imaging findings of Moyamoya disease. Stroke 2014;45:245760 CrossRef Medline

13. Xu YY, Li ML, Gao S, et al. Etiology of intracranial stenosis in young patients: a high-resolution magnetic resonance imaging study. Ann Transl Med 2017;5:319 CrossRef Medline

14. Kim DK, Verdoorn JT, Gunderson TM, et al. Comparison of noncontrast vessel wall imaging and 3D time-of-flight MRA for atherosclerotic stenosis and plaque characterization within intracranial arteries. J Neuroradiol 2020;47:266-71 CrossRef Medline

15. Xu WH, Li ML, Gao S, et al. In vivo high-resolution MR imaging of symptomatic and asymptomatic middle cerebral artery atherosclerotic stenosis. Atherosclerosis 2010;212:507-11 CrossRef Medline

16. Varnava AM, Mills PG, Davies MJ. Relationship between coronary artery remodeling and plaque vulnerability. Circulation 2002;105:93943 CrossRef Medline

17. Harteveld AA, Denswil NP, Van Hecke W, et al. Ex vivo vessel wall thickness measurements of the human circle of Willis using $7 \mathrm{~T}$ MRI. Atherosclerosis 2018;273:106-14 CrossRef Medline

18. Zhang D, Chen $\mathrm{Y}$, Chen $\mathrm{H}$, et al. A high-resolution MRI study of relationship between remodeling patterns and ischemic stroke in patients with atherosclerotic middle cerebral artery stenosis. Front Aging Neurosci 2017;9:140 CrossRef Medline

19. Ter-Karapetyan A, Triphan SM, Jobst BJ, et al. Towards quantitative perfusion MRI of the lung in COPD: the problem of short-term repeatability. PLoS One 2018;13:e0208587CrossRef Medline

20. Xu WH, Li ML, Gao S, et al. Middle cerebral artery intraplaque hemorrhage: prevalence and clinical relevance. Ann Neurol 2012;71:19598 CrossRef Medline

21. Qiao Y, Zeiler S, Mirbagheri S, et al. Intracranial plaque enhancement in patients with cerebrovascular events on high-spatial-resolution MR images. Radiology 2014;271:534-42 CrossRef Medline

22. Maaijwee NA, Rutten-Jacobs LC, Schaapsmeerders P, et al. Ischaemic stroke in young adults: risk factors and long-term consequences. Nat Rev Neurol 2014;10:315-25 CrossRef Medline 
23. Mossa-Basha M, Hwang WD, De Havenon A, et al. Multicontrast high-resolution vessel wall magnetic resonance imaging and its value in differentiating intracranial vasculopathic processes. Stroke 2015;46:1567-73 CrossRef Medline

24. Tan $H$, Chen $X$, Maingard J, et al. Intracranial vessel wall imaging with magnetic resonance imaging: current techniques and applications. World Neurosurg 2018;112:186-98 CrossRef Medline

25. O'Donnell MJ, Xavier D, Liu L, et al; INTERSTROKE investigators. Risk factors for ischaemic and intracerebral haemorrhagic stroke in 22 countries (the INTERSTROKE study): a case-control study. Lancet 2010;376:112-23 CrossRef Medline

26. Mandell DM, Matouk CC, Farb RI, et al. Vessel wall MRI to differentiate between reversible cerebral vasoconstriction syndrome and central nervous system vasculitis: preliminary results. Stroke 2012;43:860-62 CrossRef Medline
27. Ahn SH, Lee J, Kim YJ, et al. Isolated MCA disease in patients without significant atherosclerotic risk factors: a high-resolution magnetic resonance imaging study. Stroke 2015;46:697-703 CrossRef Medline

28. Obusez EC, Hui F, Hajj-Ali RA, et al. High-resolution MRI vessel wall imaging: spatial and temporal patterns of reversible cerebral vasoconstriction syndrome and central nervous system vasculitis. AJNR Am J Neuroradiol 2014;35:1527-32 CrossRef Medline

29. Van Rooij JL, Rutgers DR, Spliet WG, et al. Vessel wall enhancement on MRI in the diagnosis of primary central nervous system vasculitis. Int J Stroke 2018;13:NP24-27 CrossRef Medline

30. Sikkema T, Uyttenboogaart M, Eshghi O, et al. Intracranial artery dissection. Eur J Neurol 2014;21:820-26 CrossRef Medline

31. Li S, Yan B, Kaye A, et al. Prognosis of intracranial dissection relates to site and presenting features. J Clin Neurosci 2011;18:78993 CrossRef Medline 